\title{
Molecular screening and the clinical impacts of BCR-ABL KD mutations in patients with imatinib-resistant chronic myeloid leukemia
}

\author{
BETÜL KOÇKAN ${ }^{1}$, TAYFUR TOPTAŞ ${ }^{2}$, IŞIK ATAGÜNDÜZ ${ }^{2}$, \\ AYŞE TÜLIN TUĞLULAR ${ }^{2}$, AYŞE ÖZER $^{3}$ and MUSTAFA AKKIPRIK ${ }^{3}$ \\ ${ }^{1}$ Department of Medical Services and Techniques, Vocational School, Beykent University, \\ Buyukcekmece, 34550 Istanbul; Departments of ${ }^{2}$ Hematology and ${ }^{3}$ Medical Biology, \\ School of Medicine, Marmara University, Maltepe, 34854 Istanbul, Turkey
}

Received June 16, 2017; Accepted November 3, 2017

DOI: $10.3892 / 01.2017 .7606$

\begin{abstract}
The present study aimed to detect the frequency of kinase domain (KD) mutations in order to evaluate their clinical significance and functional importance in 45 patients with chronic myeloid leukemia (CML) who were resistant to imatinib therapy. Sanger sequencing was used (45 patients), along with allele-specific oligonucleotide polymerase chain reaction (ASO-PCR; 3 patients), for the screening of mutations. BCR/ABL KD was amplified by nested PCR and sequencing was performed. Secondly, ASO-PCR was performed to confirm the results of the sequence analysis for E255K mutations. Mutations were detected in 11/45 patients (24.44\%) via Sanger sequencing. D241G (4.4\%), C369C (4.4\%), $\mathrm{K} 285 \mathrm{~N}(2.2 \%)$, A380T (2.2\%) and A366V (2.2\%) mutations were detected. E255K (8.8\%) was detected by ASO-PCR and Sanger sequencing. Mutations are a primary reason for suboptimal responses, loss of response and resistance to imatinib. In particular, the E255K mutation, which is characterized by resistance to imatinib and nilotinib, was detected in four patients. Analyzing the mutations and monitoring patients with CML may improve their prognosis and survival rate. ASO-PCR assays will be beneficial for the routine monitoring of mutations.
\end{abstract}

Correspondence to: Professor Mustafa Akkiprik, Department of Medical Biology, School of Medicine, Marmara University, Başibüyük Mah., Maltepe Başibüyük Yolu Sok., 9/1, Maltepe, 34854 Istanbul, Turkey

E-mail: makkiprik@marmara.edu.tr

Abbreviations: CML, chronic myeloid leukemia; TKI, tyrosine kinase inhibitor; KD, kinase domain; $\mathrm{Ph}$, Philadelphia chromosome; BCR, breakpoint cluster region; ABL, Abelson; ASO-PCR, allele-specific oligonucleotide polymerase chain reaction

Key words: chronic myeloid leukaemia, breakpoint cluster region abelson kinase domain, mutation, imatinib resistance, DNA sequencing

\section{Introduction}

Chronic myelogenous leukemia (CML) is a hematopoietic stem cell disorder characterized by the Philadelphia chromosome, resulting from a $t(9 ; 22)$ reciprocal chromosomal translocation $(1,2) . t(9 ; 22)$ occurs in the juxtaposition of the Abelson (ABL1) oncogene, a tyrosine kinase (TK) located on chromosome 9, to the breakpoint cluster region (BCR) gene located on chromosome 22, leading to aberrantly increased TK activity $(3,4)$. Mechanisms that have been attributed to BCR-ABL-positive cells and been implicated in the pathogenesis of CML include increased proliferation, increased resistance to apoptosis, and an alteration of cell adhesion properties (1). The disease progresses through three phases: Chronic, accelerated and blast crisis, with disease progression likely due to an accumulation of additional genetic aberrations (5).

In May of 2001, the Food and Drug Administration approved imatinib mesylate (STI-571, Gleevec, Glivec), a selective inhibitor of the BCR-ABL TK, for the first-line treatment of patients with CML (6). Imatinib, a 2-phenyl amino pyrimidine, is a TK inhibitor (TKI) with activity against BCR-ABL. All active sites of TKs have a binding site for ATP. Imatinib mesylate works by binding close to the ATP site, locking it in a closed or self-inhibited conformation and, therefore, inhibiting the enzyme activity of the protein (7). Inhibition of BCR-ABL kinase activity by imatinib results in the transcriptional modulation of genes involved in the control of the cell cycle, cell adhesion and cytoskeleton organization (1). Imatinib functions by revoking the effects of the BCR-ABL oncoprotein through inhibiting BCR-ABL autophosphorylation and substrate phosphorylation, thus blocking proliferation and inducing apoptosis (8). Nonetheless, in certain patients, resistance to imatinib may occur. In addition to imatinib mesylate, various TKIs have recently emerged on the market, including bosutinib, dasatinib, nilotinib and ponatinib (9). These drugs are used following the failure of imatinib treatment due to resistance, disease progression or intolerance (9). Point mutations within the kinase domain (KD) of BCR-ABL, clonal chromosomal evolution, BCR-ABL amplification, pharmacogenomic 
variations, or activation of signaling shortcuts have all been implicated in imatinib mesylate resistance (10). Previous studies have reported that $\sim 20 \%$ of patients fail therapy with imatinib mesylate due to point mutations within the BCR/ABL KD (11). The emergence of point mutations in the BCR-ABL $\mathrm{KD}$ is a primary cause of imatinib resistance in patients with CML, and >90 types of KD mutations have been reported, particularly in the accelerated and blast crisis phases $(4,8,12)$. These mutations may alter the BCR-ABL KD structure and impair the imatinib-binding affinity $(4,12)$. Point mutations in the KD occur in $30-90 \%$ of patients who develop resistance to the drug (13). The sites of the KD mutations are predominantly clustered within nine amino acid positions, including T315I, Y253H/F, M351T, G250E, E255K/V, F359V and H396R, with varying sensitivities to imatinib (14). Less sensitive or resistant to BCR-ABL point mutations for dasatinib include Q252H, E255K/V, V299L, F317L, T315A and T315I; for nilotinib, they include E255K/V or F359C/V, Y253H and T315 (15). The T315I mutation was detected in $15 \%$ of 112 patients with CML who failed to respond to imatinib mesylate treatment (13). The T315I mutation is only sensitive to ponatinib (16).

To detect genetic anomalies in CML, the following methods may be used: Direct sequencing, denaturing high performance liquid chromatography (dHPLC), pyro-sequencing, ultra-deep sequencing (UDS), allele-specific oligonucleotide (ASO)-PCR (17) and denaturing gradient gel electrophoresis (DGGE) (18).

The present study was designed to determine if and what types of KD mutations were present in the 45 Turkish patients with CML enrolled, all of whom were resistant to imatinib. The aim was to detect the frequency of BCR-ABL KD mutations using a sequence analysis method to evaluate the clinical significance of the identified mutation. Furthermore, the current study intended to emphasize the importance of mutation analysis and support using the ASO-PCR method in drug selection and disease follow-up.

\section{Materials and methods}

Patients. The present study was performed retrospectively on peripheral blood (PB) samples obtained from 45 patients enrolled between January 2001 and December 2015 in the Department of Hematology at Marmara University School of Medicine (Istanbul, Turkey). In total, 45 BCR-ABL-positive patients with CML who were resistant or intolerant to imatinib were included in the present study. Patients were treated with imatinib or nilotinib or dasatinib. In total, 13 patients exhibited hematological and cytogenetic responses to imatinib, whereas 32 patients demonstrated no major molecular response and were subsequently switched to a 2nd generation TKI (nilotinib or dasatinib). Recently diagnosed chronic phase CML patients were administered imatinib (400 mg/day orally). Patients who demonstrated resistance to imatinib were administered nilotinib (100 mg/day orally) or dasatinib (100 mg/day orally). Patients had a median age of 46.6 (range, 25-87). In total, 45 patients, including 43 in the chronic phase, 1 in the accelerated phase, and 1 in blast crisis (transformed to acute myeloid leukemia), were analyzed for the presence of mutations using direct sequencing. The study protocol was approved by the Local Ethics Committee of Marmara University (Istanbul,
Turkey). Once written informed consent was obtained, $10 \mathrm{ml}$ PB samples were taken from all patients. Patients' clinical characteristics are provided in Table I.

Amplification of BCR-ABL KD by nested PCR. RNA was isolated from peripheral blood leukocytes using a 5 PRIME-Perfect Pure RNA Purification kit (Thermo Fisher Scientific Inc., Waltham, MA, USA), according to the manufacturer's protocol. cDNA was synthesized using the Bio-Speedy First Strand cDNA Synthesis kit (Bioeksen R\&D Technologies Ltd., Istanbul, Turkey) from $1 \mathrm{ng}$ total RNA in a $20 \mu \mathrm{l}$ reaction mixture, according to the manufacturer's protocol.

BCR-ABL1 transcripts were detected using nested polymerase chain reaction (PCR) using specific primers for p210 transcripts. PCR products were prepared using a pair of primers designed to cover BCR exon 13 and ABL exon 10 with the expected products of $1,643 \mathrm{bp}$. The first-round of amplification was performed using a forward primer (5'-ACA GCATTCCGCTGACCATCAATAAG-3') and a reverse primer (5'-ATGGTCCAGAGGATCGCTCTCT-3') as previously described (19). PCR reactions were performed in a final volume of $20 \mu \mathrm{l}$, containing $2 \mu \mathrm{l}$ cDNA sample, $2 \mathrm{X}$ reaction buffer, 4 mM MgSO4, 20 mM KCI, 0.4 mM dNTPs, $0.5 \mu \mathrm{M}$ each of the forward and reverse primers and $5 \mathrm{U} / \mu 1$ polymerase (Bio-Speedy Proof-Reading DNA Polymerase Pre-Mix kit; (Bioeksen R\&D Technologies Ltd.). The first step of nested PCR was conducted under touchdown PCR conditions: 2 min of initial denaturation at $95^{\circ} \mathrm{C}$, followed by a touchdown protocol of 14 cycles of $30 \mathrm{sec}$ at $95^{\circ} \mathrm{C}, 30 \mathrm{sec}$ at $67^{\circ} \mathrm{C}$ and $3 \mathrm{~min}$ at $72^{\circ} \mathrm{C}$, and then 24 cycles of $30 \mathrm{sec}$ at $95^{\circ} \mathrm{C}, 30 \mathrm{sec}$ at $60^{\circ} \mathrm{C}$ and $3 \mathrm{~min}$ at $72^{\circ} \mathrm{C}$, with a final extension for $5 \mathrm{~min}$ at $72^{\circ} \mathrm{C}(19)$.

The second round of amplification was performed using two primer pairs (Bioeksen R\&D Technologies Ltd.). Abl fragment 1 (Abl-1) amplification was performed using the following primers: Abl-1F 5'-TGGTTCATCATCATTCAA CGGTGG-3' and Abl-1R 5'-TCTGAGTGGCCATGTACA GCAGC-3' (product 447 bp, spanning codons 206-346). Abl fragment 2 (Abl-2) amplification was performed using the following primers: Abl-2F 5'-TCATGACCTACGGGAACC TC-3' and Abl-2R 5'-ATACTCCAAATGCCCAGACG-'3 (product 333 bp, spanning codons 293-428) (20). PCR reactions were performed in a total volume of $20 \mu \mathrm{l}$, containing $2 \mu \mathrm{l}$ first-round PCR product, $5 \mathrm{U} / \mu \mathrm{l}$ Taq DNA polymerase (Bioeksen R\&D Technologies Ltd.), 2X Phusion buffer, $4 \mathrm{mM}$ MgSO4, $20 \mathrm{mM} \mathrm{KCI}, 0.4 \mathrm{mM}$ dNTPs and $0.5 \mu \mathrm{M}$ of each primer.

Conditions for the second step were as follows: $30 \mathrm{sec}$ of initial denaturation at $98^{\circ} \mathrm{C}$, amplification for 40 cycles of $10 \mathrm{sec}$ at $98^{\circ} \mathrm{C}, 30 \mathrm{sec}$ at $60^{\circ} \mathrm{C}$ and $40 \mathrm{sec}$ at $72^{\circ} \mathrm{C}$, with a final extension step of $5 \mathrm{~min}$ at $72^{\circ} \mathrm{C}$ (4). The nested PCR products (5 $\mu$ l loaded per well) were then resolved via $2 \%$ agarose gel electrophoresis and visualized using ethidium bromide. Quantification of PCR bands densitometry was performed using ImageJ 1.41o software (National Institutes of Health, Bethesda, MD, USA).

BCR-ABL KD mutations screening by Sanger sequencing. PCR products from nested PCR reactions were analyzed by Sanger sequencing. The PCR products were sent to Macrogen 
Europe (Amsterdam, The Netherlands) for Sanger sequencing. ABL fragment 1 (spanning codons 206-346) and ABL fragment 2 (spanning codons 293-428) of BCR-ABL KD were sequenced. The results were compared with the ABL-1 sequence (GenBank: EU216071.1).

ASO-PCR assay for E255K mutations. Genomic DNA (gDNA) was extracted from peripheral blood samples using a MagNA Pure LC DNA Isolation kit (Roche Molecular Diagnostics, Pleasanton, CA, USA). The quantity and ratio of absorbance at 260 and $280 \mathrm{~nm}$ (A260/280) of the purified gDNA was determined with a Qubit ${ }^{\circledR}$ Fluorometer (Thermo Fisher Scientific, Inc.).

BCR-ABL transcripts were detected in three patients by ASO-PCR using allele-specific primers for p210, according to the E255K mutation. To amplify the E255K mutation, the forward 5'-GCGGGGGCCAGTACGGGA-3' and reverse 5'-GCCAATGAAGCCCTCGGAC-3' primers were used, as previously described (21). The thermocycling conditions used were as follows: $5 \mathrm{~min}$ at $94^{\circ} \mathrm{C}$ followed by 35 cycles of denaturation at $94^{\circ} \mathrm{C}$ for $30 \mathrm{sec}$, annealing at $60^{\circ} \mathrm{C}$ for $30 \mathrm{sec}$, extension at $72^{\circ} \mathrm{C}$ for $45 \mathrm{sec}$, and a final extension for $5 \mathrm{~min}$ at $72^{\circ} \mathrm{C}$. The ASO-PCR products ( $5 \mu 1$ loaded per well) then resolved via $2 \%$ agarose gel electrophoresis and visualized with ethidium bromide. One peripheral blood sample was obtained from a patient with CML exhibiting wild type for ABL mutations and used as a negative control. Quantification of PCR bands densitometry was performed using ImageJ 1.41o software (National Institutes of Health). Results were confirmed using sequencing analysis.

Statistical analysis. Statistical data were analyzed using GraphPad Prism 6.0 (GraphPad Software, Inc., La Jolla, CA, USA). The associations between BCR-ABL KD mutations and the clinical parameters were determined using Fisher's exact test, and an unpaired t-test when required. All tests were two-tailed, and $\mathrm{P}<0.05$ was considered to indicate a statistically significant difference.

\section{Results}

Frequency of BCR-ABL KD mutations. The six types of ABL KD mutations detected by Sanger sequencing in the present study are demonstrated in Fig. 1. In total, 11/45 (24.44\%) patients had mutations that were detected by Sanger sequencing. An E255K mutation was detected in 4 patients $(8.8 \%)$, D241G mutation was detected in 2 patients $(4.4 \%)$, C369C mutation was detected in 2 patients $(4.4 \%), \mathrm{K} 285 \mathrm{~N}$ mutation was detected in 1 patient $(2.2 \%)$, A380T mutation was detected in 1 patient $(2.2 \%)$ and an A366V mutation was detected in 1 patient (2.2\%) (Table II). There was no significant association between age, sex, median white blood cell count, platelet count and haemoglobin count, International Scale value (16) and mutation (Table I).

Verification of the E255K mutation using ASO-PCR. Sequencing data confirmed the presence of an E255K mutation in three previously Sanger sequencing-detected patients, using ASO-PCR. Of note, one patient with the E255K mutation succumbed to CML due to disease progression during the
Table I. Clinical characteristics of patients with CML carrying MT and WT ABL KDs.

\begin{tabular}{lccc}
\hline Characteristics & $\begin{array}{c}\text { Mutant } \\
\text { type }\end{array}$ & $\begin{array}{c}\text { Wild } \\
\text { type }\end{array}$ & P-value \\
\hline Sex & & & \\
$\quad$ Male & 6 & 15 & $\mathrm{NS}$ \\
Female & 5 & 10 & \\
Median age, years & 48 & 50 & 0.94 \\
Median WBC counts $\left(\mathrm{x} 10^{9} / \mathrm{l}\right)^{\mathrm{a}}$ & 6.000 & 5.650 & 0.41 \\
Median PLT counts $\left(\mathrm{x} 10^{9} / \mathrm{l}\right)^{\mathrm{a}}$ & 245.000 & 224.000 & 0.42 \\
Median Hb $(\mathrm{g} / \mathrm{dl})^{\mathrm{a}}$ & 12 & 12.25 & 0.52 \\
IS at mutation detection $^{\mathrm{a}}$ & 4.43 & 0.47 & 0.85 \\
\hline
\end{tabular}

CML, chronic myeloid leukemia; MT, mutant; WT, wild type; WBC, white blood cell; PLT, platelet, Hb, hemoglobin; IS, international scale.

study, thus ASO-PCR could not be performed for this patient. Fig. 2 presents the monitoring data (obtained from electrophoresis results) for the detected $\mathrm{E} 255 \mathrm{~K}$ mutation. DNA molecules were visualized using ultraviolet light following staining with ethidium bromide.

\section{Discussion}

Imatinib mesylate is a first line therapy for patients with CML, a selective inhibitor of the BCR/ABL TK (6). Point mutations within the KD of BCR-ABL have been previously associated with promoting drug resistance. Furthermore, additional resistance mechanisms including; clonal chromosomal evolution, BCR-ABL amplification, pharmacogenomic variations are present in patients with and without kinase domain mutations (10). However, the exact contribution(s) to resistance are yet to be determined. Additionally, the inconsistent use of prescription drugs by patients results in resistance development (10).

Unidentified mutations in a given sequence may be detected using methods such as direct sequencing, DGGE and dHPLC; previously recognized mutations can also be identified with methods including ASO-PCR and RFLP. Direct sequencing is the most widespread method applied in the routine monitoring of patients, but it has limited sensitivity (10-25\%). DGGE is a powerful technique, which may detect single base mutations up to $100 \%$ sensitivity; however, its sensitivity decreases for fragments larger than $500 \mathrm{bp}$, requiring the use of specialized equipment. dHPLC is a simple method for detecting low-level mutations which is more sensitive when compared with direct sequencing, and it may recognize sequence variations (17). However, dHPLC is not as common as direct sequencing and it may generate false negative results in cases with a vast number of mutant subclones. ASO-PCR has been designed to detect specific mutations; therefore, it is a troublesome method to use in screening for more than one mutation, and is therefore not used frequently (17). In a previous study that used the dHPLC method in Turkey (22), 8 types of mutations were detected, all of which are different from the 6 types of mutations detected 
Table II. Sanger-sequencing detected mutations and their properties.

\begin{tabular}{|c|c|c|c|c|c|c|}
\hline Patient code & Mutation & $\begin{array}{c}\text { Amino acid } \\
\text { changes }\end{array}$ & $\begin{array}{c}\mathrm{BCR} / \mathrm{ABL} \\
\% \text { (IS) }\end{array}$ & $\begin{array}{c}\mathrm{BCR} / \mathrm{ABL} \\
\text { type }\end{array}$ & Exon & Treatment $^{\mathrm{a}}$ \\
\hline 3 & D241G (heterozygous) & GACàGGC (A>G) & 42.9 & $\mathrm{p} 210$ & Exon 4 (p-loop) & Imatinib \\
\hline 4 & E255K (homozygous) & GAGàAAG $(G>A)$ & 0.151 & p210 & Exon 4 (p-loop) & Glivec \\
\hline 5 & E255K (homozygous) & GAGàAAG $(G>A)$ & 4.43 & p210 & Exon 4 (p-loop) & Nilotinib \\
\hline 7 & E255K (homozygous) & GAGàAAG $(G>A)$ & 0.81 & p210 & Exon 4 (p-loop) & Imatinib \\
\hline 9 & D241G (heterozygous) & GACàGGC (A>G) & 8.35 & p210 & Exon 4 (p-loop) & Dasatinib \\
\hline 10 & E255K (homozygous) & GAGàAAG $(G>A)$ & 38.4 & p210 & Exon 4 (p-loop) & Nilotinib \\
\hline 28 & K285N (homozygous) & AAAàAAT (A>T) & 0.362 & p210 & Exon 5 (IM B.S.) & Imavec \\
\hline 14 & C369C (heterozygous) & TGCà TGT (C>T) & 0.0013 & p210 & Exon 7 (C-loop) & Dasatinib \\
\hline 24 & A380T (heterozygous) & GCTàACT (G>A) & 0.15 & p210 & Exon 7 (A-loop) & Dasatinib \\
\hline 15 & C369C (heterozygous) & TGCà TGT (C>T) & 0.02 & p210 & Exon 7 (C-loop) & Dasatinib \\
\hline 40 & A366V (heterozygous) & GCCàGTC (C>T) & 0.003 & p210 & Exon 7 (C-loop) & Nilotinib \\
\hline
\end{tabular}

${ }^{a}$ Used TKI after mutation detection. IS, international scale at mutation detection; BCR, breakpoint cluster region; ABL, Abelson.

A cGCACGgacatcaccat

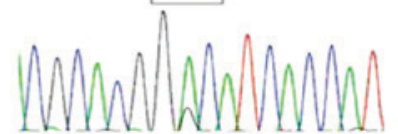

B GTACGgGAAGgTGTAC

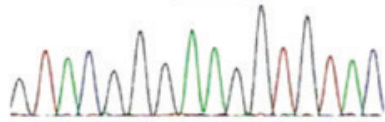

C CGAAACTGCCTGGTAG

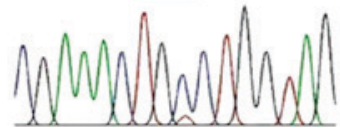

D ACTTGgtgaAgGtaACTGATT

GAC>GGC: D241G

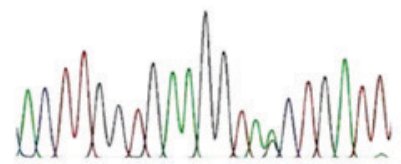

E

TTGAATGAAGCTGC

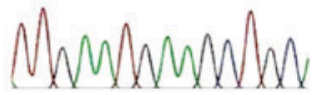

$\mathbf{F}$

\section{TTGCTGCCCGAA}

TGC>TGT: C369C
AAA>AAT: $K 285 N$

GCT>ACT: A380T

GCC>GTC: A366V

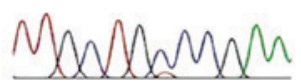

Figure 1. Sequencing results of BCR/ABL KD mutations in six patients. (A) $A>G$ (D241G); (B) G>A (E255K); (C) C>T (C369C); (D) G>A (A380T); (E) A>T (K285N); (F) C>T (A366V). BCR, breakpoint cluster region; ABL, Abelson; KD, kinase domain.

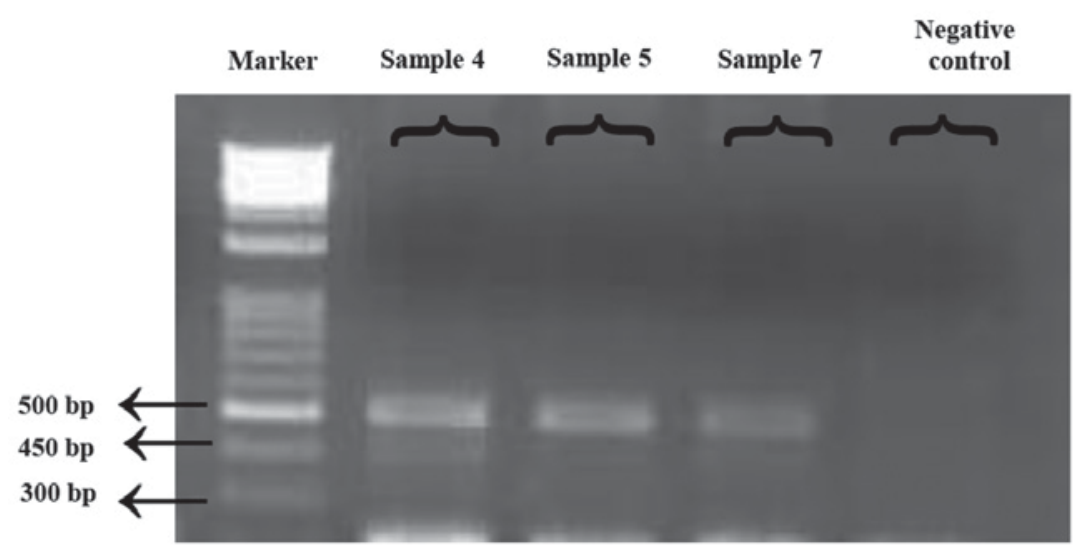

Figure 2. Data on the detected E255K mutations in four patients. For the detected mutations, ASO-PCR products resolved via agarose gel electrophoresis are presented. Marker, DNA ladder $100 \mathrm{bp}$. Sample 4: PCR-amplified product of the E255K mutation using gDNA from a patient with CML receiving glivec treatment. Sample 5: PCR-amplified product of the E255K mutation using gDNA from a patient with CML receiving nilotinib treatment. Sample 7: PCR-amplified product of the E255K mutation using gDNA from a patient with CML receiving imatinib treatment. Negative control: One peripheral blood sample was obtained from a patient with CML exihibiting wild type for ABL mutations and used as a negative control. ASO-PCR, allele-specific oligonucleotide polymerase chain reaction; CML, chronic myeloid leukemia; gDNA, genomic DNA. 
in the present study. As ASO-PCR primers should be specifically designed for each individual mutation, ASO-PCR may be considered a troublesome method in this respect. The use of the ASO-PCR method may be advantageous for detecting the most common mutations in the disease, as ASO-PCR assays have the best sensitivity for detecting mutations even following dilutions in the 10,000-fold range (23). ASO-PCR is also a more sensitive method when compared with dHPLC and direct sequencing. ASO-PCR assays have various advantages, including quicker analysis results, lower costs and an easy protocol (23). For this reason, an ASO-PCR assay was used in the present study for detecting the E255K mutation; the results of ASO-PCR mutation analysis were compared with those from direct sequencing. The current study focused on the frequency and types of KD mutations in imatinib-resistant Turkish patients with CML, and described the occurrence of mutations in the BCR/ABL KD in $11 / 45$ patients with CML, detected by Sanger sequencing. Using two different methodological approaches, namely Sanger sequencing and ASO-PCR, the E255K mutation was identified in 4/45 patients with CML.

The BCR/ABL KD mutation frequency was $24.44 \%$ $(11 / 45)$ in the present study. Compared with the literature, it was observed that the frequency of BCR/ABL KD mutations $(24.44 \%)$ present in the current study was slightly lower compared with that stated in previous studies, which reported the overall incidence of KD mutations as $33 \%$ in Thailand (4), 44\% in Korea (23), 41.53\% in India (24) and 30\% in Germany (25); however, it was slightly increased compared with, though similar to, the previously recorded $23 \%$ in a Turkish population (22).

In the present study, 6 diverse types of mutations were detected, including E255K, D241G, C369C, K285N, A380T and A366V. Erbilgin et al (22) detected 8 types of mutations, including M244V, G250E, Y253H, V304I, F359C, K357R, V304I and K357R. The mutations detected in these two studies were all distinct from each other, demonstrating that there is a wide spectrum of ABL KD region mutations in the Turkish population of patients with CML.

The most resistant mutation T315I could not be detected in any of the 45 patients with CML in the present study; therefore, the results are not concordant with the literature with respect to the $\mathrm{T} 315 \mathrm{I}$ mutation. The E255K mutation is located in exon 4 (P-loop region) on ABL, as confirmed by the My Cancer Genome database (https://www.mycancergenome. org). The E255K mutation results in an amino acid substitution at position 255 in BCR-ABL, from a glutamate to a lysine. The E255K mutation frequency was determined to be $8.8 \%$. In the OncQuest and GIMEMA studies, the E255K mutation frequency was reported to be 6.9 and $16.5 \%$, respectively. With respect to the $\mathrm{E} 255 \mathrm{~K}$ mutation, the results of the present study are consistent with previous literature. The E255K mutation has been associated with imatinib and nilotinib resistance in patients with CML (8). Dasatinib therapy is recommended in the presence of an E255K mutation, according to the European LeukemiaNet recommendations (8).

D241G point mutations, targeting amino acids located near the P-loop, have been previously described (26). A366V mutations (substitution) at the C-loop have also been described in a prior study (27) and confirmed by the Catalogue of Somatic Mutations in Cancer database (http://cancer.sanger.ac.uk/cosmic). According to localization, these mutations may be associated with drug resistance and could affect the binding mechanisms, but further analysis is required for confirmation. The remaining four mutations defined in the current study have been previously described in association with imatinib mesylate resistance (8). The K285N mutation was determined to confer a lower imatinib binding affinity. In a previous in vitro trial, it was demonstrated that $\mathrm{K} 285 \mathrm{~N}$ mutants had a lower binding affinity for imatinib when compared with the native type (28), and exhibited resistance to imatinib (29). The A380T mutation was detected at the A-loop in one patient, who received dasatinib treatment due to imatinib-resistance. The A380T mutation was previously detected as an ABL kinase mutation in imatinib-resistant patients with CML $(30,31)$. Additionally, C369C is available in the Catalogue of Somatic Mutations in Cancer database (http://cancer.sanger.ac.uk/cosmic). It is a silent mutation; therefore, it has not been discussed in the literature to the best of our knowledge.

ASO-PCR experiments were performed using forward and reverse primers, and reliable results were obtained via these means. The ASO-PCR assay exhibits numerous advantages including an increase in sensitivity, short analysis time, lower costs, and a simple procedure. Kang et al (23) previously identified 11 types of mutations with a $97 \%$ sensitivity using the ASO-PCR method. Iqbal et al (21) identified 4 different types of mutations using the ASO-PCR method. Thus, it was concluded that the ASO-PCR method is a reliable tool for routine research.

By determining the mutational profile of patients, the optimal treatment (2nd generation TKIs, alloSCT, etc.) for each patient may be selected. The ASO-PCR assay has a high sensitivity for the identified mutations. The results suggest that using ASO-PCR assays will be beneficial in the routine monitoring of mutations, particularly for commonly identified mutations in patients with CML.

\section{Acknowledgements}

The present study was supported by the Scientific Research Projects Committee (grant no. SAG-C-YLP-110315-0057) at Marmara University.

\section{References}

1. Deininger MW, Goldman JM and Melo JV: The molecular biology of chronic myeloid leukemia. Blood 96: 3343-3356, 2000

2. Corbin AS, La Rosée P, Stoffregen EP, Druker BJ and Deininger MW: Several Bcr-Abl kinase domain mutants associated with imatinib mesylate resistance remain sensitive to imatinib. Blood 101: 4611-4614, 2003.

3. Litzow MR: Imatinib resistance, obstacles and opportunities. Arch Pathol Lab Med 130: 669-679, 2006.

4. Wongboonma W, Thongnoppakhun W and Auewarakul CU: BCR-ABL kinase domain mutations in tyrosine kinase inhibitors-naïve and -exposed Southeast Asian chronic myeloid leukemia patients. Exp Mol Pathol 92: 259-265, 2012.

5. Sawyers CL: Chronic myeloid leukemia. N Engl J Med 340: 1330-1340, 1999.

6. O'Brien S, Berman E, Borghaei H, DeAngelo DJ, Devetten MP, Devine S, Erba HP, Gotlib J, Jagasia M, Moore JO, et al: NCCN clinical practice guidelines in oncology: Chronic myelogenous leukemia. J Natl Compr Canc Netw 7: 984-1023, 2009.

7. Iqbal $\mathrm{N}$ and Iqbal $\mathrm{N}$ : Imatinib: A breakthrough of targeted therapy in cancer. Chemother Res Pract 2014: 357027, 2014. 
8. Soverini S, Hochhaus A, Nicolini FE, Gruber F, Lange T, Saglio G, Pane F, Müller MC, Ernst T, Rosti G, et al: BCR-ABL kinase domain mutation analysis in chronic myeloid leukemia patients treated with tyrosine kinase inhibitors: Recommendations from an expert panel on behalf of European Leukemia net. Blood 118: 1208-1215, 2011.

9. Ursan ID, Jiang R, Pickard EM, Lee TA, Ng D and Pickard AS: Emergence of BCR-ABL kinase domain mutations associated with newly diagnosed chronic myeloid leukemia: A meta-analysis of clinical trials of tyrosine kinase inhibitors. J Manag Care Spec Pharm 21: 114-122, 2015.

10. La Rosée $P$ and Deininger MW: Resistance to imatinib: Mutations and beyond. Semin Hematol. 47: 335-343, 2010.

11. Vaidya S, Vundinti BR, Shanmukhaiah C, Chakrabarti P and Ghosh K: Evolution of BCR/ABL gene mutation in CML is time dependent and dependent on the pressure exerted by tyrosine kinase inhibitor. PLoS One 10: e0114828, 2015.

12. Wang Z, Liu Z, Wu X, Chu S, Wang J, Yuan H, Roth M, Yuan YC, Bhatia R and Chen WY: Correction: ATRA-induced cellular differentiation and CD38 expression inhibits acquisition of BCR-ABL mutations for CML acquired resistance. PLoS Genet 10: e1004414, 2014.

13. Cortes J, Jabbour E, Kantarjian H, Yin CC, Shan J, O'Brien S, Garcia-Manero G, Giles F, Breeden M, Reeves N et al: Dynamics of BCR-ABL kinase domain mutations in chronic myeloid leukemia after sequential treatment with multiple tyrosine kinase inhibitors. Blood 110: 4005-4011, 2007.

14. Apperley JF: Part I: Mechanisms of resistance to imatinib in chronic myeloid leukaemia. Lancet Oncol 8: 1018-1029, 2007.

15. Jabbour E, Hochhaus A, Cortes J, La Rosée P and Kantarjian HM Choosing the best treatment strategy for chronic myeloid leukemia patients resistant to imatinib: Weighing the efficacy and safety of individual drugs with BCR-ABL mutations and patient history. Leukemia 24: 6-12, 2010.

16. Baccarani M, Castagnetti F, Gugliotta G and Rosti G: A review of the european leukemianet recommendations for the management of CML. Ann Hematol 94 (Suppl 2): S141-S147, 2015.

17. Ai J and Tiu RV: Practical management of patients with chronic myeloid leukemia who develop tyrosine kinase inhibitor-resistan BCR-ABL1 mutations. Ther Adv Hematol 5: 107-120, 2014

18. Sorel N, Roy L, Martineau G, Guilhot F, Turhan AG and Chomel JC: Sequential emergence of ABL-kinase mutations with loss of unmutated BCR-ABL allele during targeted therapies of CML. Blood 108: 1782-1783, 2006.

19. Ernst T, Erben P, Muller MC, Paschka P, Schenk T, Hoffmann J, Kreil S, La Rosée P, Hehlmann R and Hochhaus A: Dynamics of BCR-ABL mutated clones prior to hematologic or cytogenetic resistance to imatinib. Haematologica 93: 186-192, 2008.

20. Deininger MW, McGreevey L, Willis S, Bainbridge TM Druker BJ and Heinrich MC: Detection of ABL kinase domain mutations with denaturing high performance liquid chromatography. Leukemia 18: 864-871, 2004
21. Iqbal Z, Aleem A, Iqbal M, Naqvi MI, Gill A, Taj AS, Qayyum A, ur-Rehman N, Khalid AM, Shah IH, et al: Sensitive detection of pre-existing BCR-ABL kinase domain mutations in CD34+ cells of newly diagnosed chronic-phase chronic myeloid leukemia patients is associated with imatinib resistance: Implications in the post-imatinib era. PLoS One 8: e55717, 2013.

22. Erbilgin Y, Catal S, Eskazan AE, Hatirnaz Ö, Soysal T and Özbek U: ABL gene kinase domain mutation scanning by denaturing high performance liquid chromatography sequencing method. Turk J Hematol 28: 97-102, 2011.

23. Kang HY, Hwang JY, Kim SH, Goh HG, Kim M and Kim DW: Comparison of allele specific oligonucleotide-polymerase chain reaction and direct sequencing for high throughput screening of ABL kinase domain mutations in chronic myeloid leukemia resistant to imatinib. Haematologica 91: 659-662, 2006.

24. Srivastava S and Dutt S: Imatinib mesylate resistance and mutations: An Indian experience. Indian J Med Paediatr Oncol 34: 213-220, 2013

25. Sherbenou DW, Hantschel O, Kaupe I, Willis S, Bumm T, Turaga LP, Lange T, Dao K, Press RD, Druker BJ, et al: BCR-ABL SH3-SH2 domain mutations in chronic myeloid leukemia patients on imatinib. Blood 116: 3278-3285, 2010.

26. Gruber F, Hansen H, Olsen M, Eggen L, Skogen B, Gedde-Dahl T, Lundan T, Porkka K, Simonsson B, Markevärn B, et al: Quantitative analyses of BCR-ABL mutations associated with imatinib treatment in CML. Blood 106: 2000, 2005.

27. Khorashad JS, Kelley TW, Szankasi P, Mason CC, Soverini S, AdriaL T, Eide CA, Zabriskie MS, Lange T, Estrada JC, et al: BCR-ABL1 compound mutations in tyrosine kinase inhibitor-resistant CML: Frequency and clonal relationships. Blood 121: 489-498, 2013

28. Rajasekaran R, Doss PDC, Prasad GA and Sethumadhavan R: In silico identification and analysis of drug resistant mutants of ABL tyrosine kinase based on detrimental missense mutations. Curr Signal Trans Ther 6: 396-404, 2011.

29. von Bubnoff N, Veach DR, van der Kuip H, Aulitzky WE, Sänger J, Seipel P, Bornmann WG, Peschel C, Clarkson B and Duyster J: A cell-based screen for resistance of Bcr-Abl-positive leukemia identifies the mutation pattern for PD166326, an alternative Abl kinase inhibitor. Blood 105: 1652-1659, 2005.

30. Soverini S, Baccarani M, Iacobucci I and Martinelli G: Resistance to tyrosine kinase inhibitors in Philadelphia chromosome-positive leukemias: Which mutations matter? Clinical Leukemia 1: 223-228, 2007.

31. Chomel JC, Sorel N and Turhan AG: ABL-Kinase mutations in progenitors and stem cells from chronic myeloid leukemia patients. Stem Cells Cancer Stem Cells 8: 305-315, 2012. 